\title{
A Modified Approach Below the Lateral Arcuate Ligament to Facilitate the Subcostal Anterior Quadratus Lumborum Block
}

This article was published in the following Dove Press journal:

Journal of Pain Research

\section{Huili Li (D) \\ Rong Shi \\ Yun Wang (ID}

Department of Anesthesiology, Beijing Chaoyang Hospital, Capital Medical University, Beijing, 100020, People's Republic of China
Correspondence: Yun Wang Department of Anesthesiology, Beijing Chaoyang Hospital, Capital Medical University, Beijing, 100020, People's Republic of China

Tel +86-10-85231330

Email wangyun129@ccmu.edu.cn
Purpose: The subcostal quadratus lumborum (QL) block was used in postoperative analgesia for abdominal surgery. However, it is difficult to precisely put the needle tip into the target fascia compartment. In the current study, we proposed a modified approach to facilitate the subcostal QL block.

Patients and Methods: Twenty-four patients scheduled for laparoscopic renal surgery were enrolled. The modified QL block was placed preoperatively. The transducer was placed just laterally to the tip of L1 transverse process to perform the parasagittal scan. The needle was inserted in-plane and advanced toward the target compartment between the QL muscle and the anterior thoracolumbar fascia and just below the lateral arcuate ligament. The $20 \mathrm{~mL}$ of $0.5 \%$ ropivacaine was injected slowly if the saline spread cranially via the posterior pathway of lateral arcuate ligament was observed on the sonogram. Then, the paramedian transverse scanning at the level of T12-L1 was performed to observe the injectate diffusion. The dermatomal coverage of sensory block was tested at $5 \mathrm{~min}$ and $10 \mathrm{~min}$ after LA injections. The complications associated with the block were recorded.

Results: Twenty-three (95.8\%) patients received the successful block. All patients achieved the sensory block dermatomes of T9-T12 and T6-L1, at 5 and 10 minutes after injection, respectively. The mean numbers of block dermatomes were $5.6 \pm 1.8$ at $5 \mathrm{~min}$ and $6.5 \pm 2.2$ at $10 \mathrm{~min}$ after injection. Two out of twenty-three (8.7\%) patients achieved coverage as cephalad as T5 at 10 min after injections. The lunar-shaped spread of LA along the diaphragm into the T12 paravertebral space was observed in every patient. No complications occurred.

Conclusion: The modified subcostal QL block has the advantages of clear sonoanatomy, rapid onset time, and consistent dermatomal coverage and provides a new choice for postoperative analgesia of abdominal surgery.

Trial Registration: Chinese Clinical Trial Registry: ChiCTR2000029210.

Keywords: quadratus lumborum block, ultrasound, diaphragm, arcuate ligament

\section{Introduction}

The thoracolumbar fascia provides a retinaculum for the paraspinal and posterolateral abdominal wall muscles. In the three-layered model, the posterior thoracolumbar fascia layer surrounds the erector spinae muscles, the middle layer passes between the erector spinae muscles and quadratus lumborum $(\mathrm{QL})$, and the anterior layer lies anterior to both $\mathrm{QL}$ and psoas muscles. In the two-layered model, the fascia on the anterior aspect of the QL muscle is referred to as the transversalis fascia. Cranially, the anterior thoracolumbar fascia (ATF) divides into two layers. 
One layer is continuous with the endothoracic fascia in the thorax, and the other layer blends with the diaphragm at the arcuate ligaments.

The classic subcostal QL block targets the local anesthetic (LA) between the investing fascia of QL and the ATF. ${ }^{1}$ The injected LA can spread cranially under the latera arcuate ligament to the lower thoracic paravertebral space posterior to the endothoracic fascia, which has been validated as the action mechanism in a cadaver study. ${ }^{2,3}$ However, it is frequently difficult for anaesthetists to distinguish the ATF from the investing fascia of QL during ultrasound scanning, especially in obese or elderly patients. In addition, the ATF may fuse with the posterior lamina of the posterior renal fascia and the blending site varies between patients. ${ }^{4,5}$ Together, the multilaminated fascial structures anterior to the QL indicate the precision injection is of great difficult in the classic subcostal QL block. Based on the action mechanism of classic subcostal QL block, we think that it indicates the correct position of needle endpoint if the cranial spread of injected LA via the posterior pathway of lateral arcuate ligament could be confirmed under the ultrasound visualization. In other words, the cranial spread of LA via the posterior pathway of lateral arcuate ligament can be considered as an indicator of successful subcostal QL block. However, in the classic subcostal QL block, the probe is placed too laterally to clearly visualize the diaphragm and lateral arcuate ligament. Therefore, we proposed a modified subcostal QL block below the lateral arcuate ligament by replacing the probe over to the tip of L1 transverse process (TP) and performing a sagittal scan. In our modified approach, the acoustic shadows of T12 rib and the apposition zone (the posterior pathway of lateral arcuate ligament) between the QL and diaphragm were visualized and the cranial spread of LA via the posterior pathway of the lateral arcuate ligament could also be identified under ultrasound guidance.

\section{Methods}

This study followed the Declaration of Helsinki. Ethical approval for this study (2019-ke-369) was provided by the Ethical Committee of Beijing Chaoyang Hospital, Capital Medical University, China (Chairperson Prof. Yong Chen) on 6 January, 2020. This study was registered with the Chinese Clinical Trial Registry, clinical trial number ChiCTR2000029220, date of registration 19 January, 2020. After the written informed consent was obtained, twenty-four patients American Society of Anesthesiologists physical status (ASA) I-III scheduled for laparoscopic renal surgery were enrolled for this study, in whom general anesthesia combined with the modified subcostal QL block was planned. Patients were excluded if they had a body mass index $(\mathrm{BMI})>28$ and more than $70 \mathrm{yr}$, clinically known spinal deformity, infection in the back, allergy to LA, or coagulopathy.

The block was placed before the induction of general anesthesia. After the patient arrived in the operating theatre, intravenous access was established, and patient vitals including heart rate, arterial pressure, arterial oxygen saturation, and electrocardiography were monitored. The patients were positioned in the prone position. All ultrasound scans were performed using an ultrasound system and a curved array probe (C5-2, 5-2 $\mathrm{MHz}$ frequency, KONICA MINOLTA, Tokyo, Japan). A right-hand dominant anesthetist (Dr. Yun Wang) experienced in ultrasound imaging and interventional injections conducted the ultrasound scanning protocols and the modified subcostal QL block below the lateral arcuate ligament. The probe was held in the non-dominant hand of the operator and was positioned $6-8 \mathrm{~cm}$ lateral to the spine midline, with its orientation marker directed cranially. The QL was identified as a long curve muscle with cranial end attached to the T12 rib. Then, the probe was moved medially until the tip of L1 TP appeared after the acoustic shadow of T12 rib was identified, while still maintaining the same orientation. Finally, the probe was positioned over the tip of L1 TP on the affected side to perform a sagittal scan. The acoustic shadows of T12 rib and the apposition zone (the posterior pathway of lateral arcuate ligament) between the QL and diaphragm were visualized in the sonogram (Figure 1). The ultrasound scout scan was deemed successful if the moving diaphragm, the QL muscle and the continuity of lateral arcuate ligament with the ATF were visualized. Those patients with successful ultrasound scout scan underwent the modified QL block below the lateral arcuate ligament. The position of the probe was then marked on the patient's back using a skin marking pen (Figure 2A).

Following proper sterile technique, the probe and cable were covered with a sterile plastic sleeve. Iodophor served as the coupling agent. While this strategy did slightly reduce overall ultrasound image quality, proper adjustments to gain and compression settings allowed for effective image quality optimization. ${ }^{6,7}$ To assess the ultrasound visibility of the diaphragm, the QL muscle and the ATF, an expert sonographer who was blinded as to the ultrasound-guided injections 


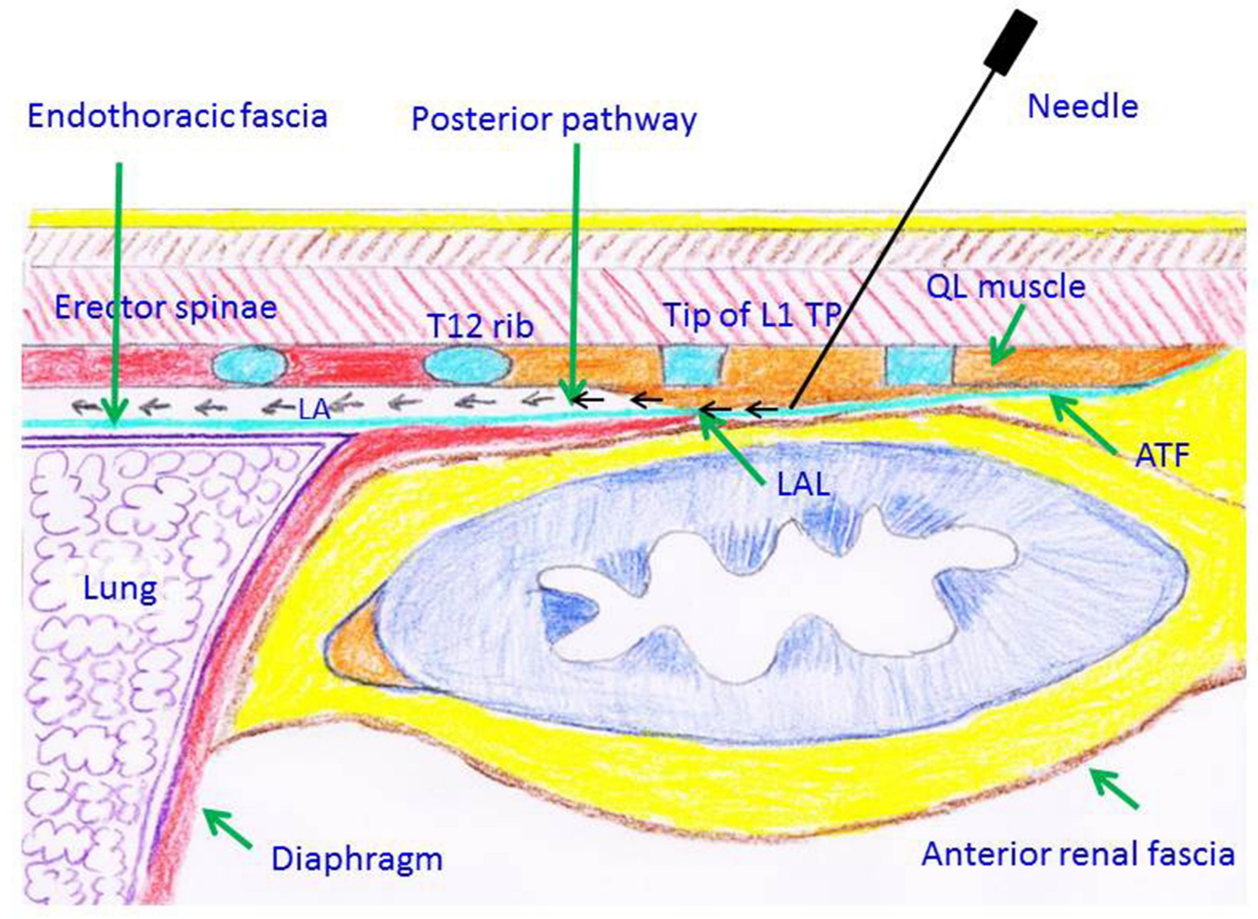

Figure I The Schematic diagram describing the action mechanism of the modified subcostal QL block (parasagittal view through the tip of LI TP). The ATF is divided into two leaflets when it approaches the LAL, and the upper leaflet is in continuity with the endothoracic fascia. The lower leaflet blends with the LAL. The LA spreads cranially via a posterior pathway to the LAL and into lower thoracic paravertebral space posterior to the endothoracic fascia after the modified subcostal QL block below the LAL (black arrows).

Abbreviations: TP, transverse process; QL, quadratus lumborum; LAL, lateral arcuate ligament; LA, local anesthetic; ATF, anterior thoracolumbar fascia.

used a 4-point scoring system to assess the visibility of them ( 0 , not visible; 1 , barely visible; 2 , clearly visible; 3 , very clearly visible). The total ultrasound visibility score of the three structures was determined for every patient. The total ultrasound visibility of the three structures was determined to be good if the mean total visibility score was $>6$, average if the score was $3-6$, and poor if the score was $<3$. 6,8

2-3 $\mathrm{mL}$ of $1 \%$ lidocaine was injected into the skin $2 \mathrm{~cm}$ caudal to the ultrasound probe for local infiltration. A $18 \mathrm{G}$ puncture needle (TUOREN, Henan, China) was next inserted in the plane of the ultrasound beam such that its tip was oriented towards the target injection site below the lateral arcuate ligament and between the ATF and the investing fascia of QL muscle (Figure 1). The needle angle was optimized while in the erector spinae muscle, and the needle was then slowly advanced into the target compartment. The hydro-dissection was performed by injecting $0.9 \%$ saline after careful aspiration to avoid inadvertent administration of LA into vessels. The $20 \mathrm{~mL}$ of $0.5 \%$ ropivacaine was injected slowly if the saline spread cranially via the posterior pathway of lateral arcuate ligament was observed on the sonogram. Otherwise, the depth of needle tip was slightly adjusted till the cranial spread of saline via the posterior pathway of lateral arcuate ligament was observed. Then, the paramedian transverse scanning at the level of T12-L1 was performed immediately to observe the diffusion of injected LA. The spread pattern of LA was recorded.

Patients were then repositioned to a supine position. The dermatomal coverage of sensory blockade was then tested at $5 \mathrm{~min}$ and $10 \mathrm{~min}$ after the LA injections. The reduced sensation to an ice block was identified as sensory block. The assistant who tested the dermatomal coverage of sensory blockade was blinded as to the ultrasoundguided injections. Then, anesthesia induction and tracheal intubation were performed in all patients.

The number of attempts, as defined by the number of times in which direction was adjusted without altering the puncture site, was also recorded. The procedure duration was recorded from the scanning of structures after proper sterile technique to the time of LA injection completion, excluded time to assess the structures visibility. At 2 $\mathrm{h}$ after the operation, an expert sonographer evaluated the occurrence of postoperative complications such as local 

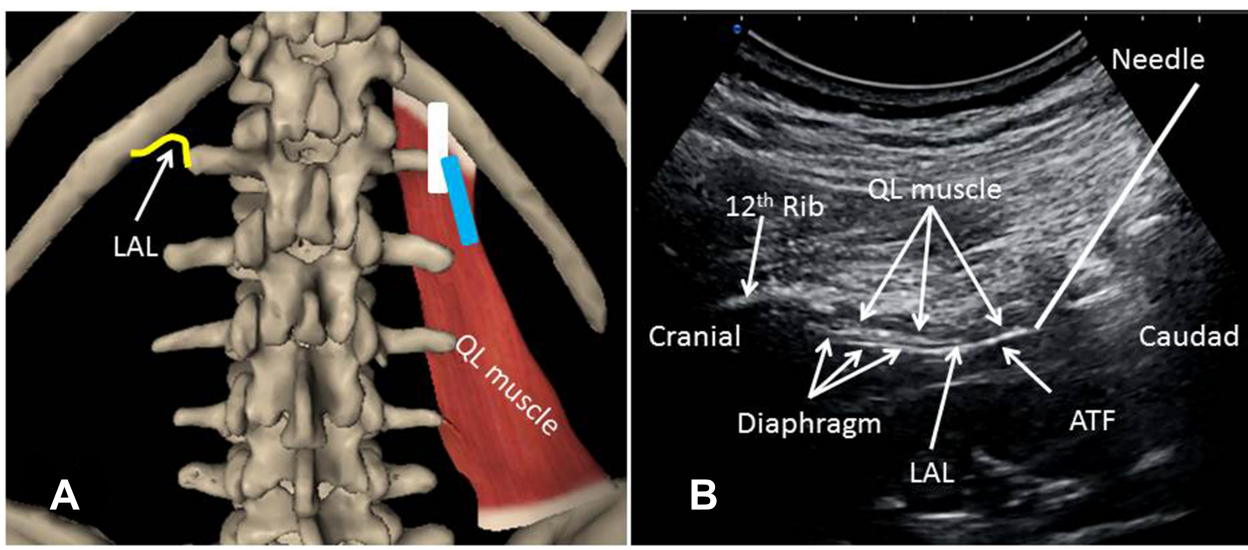

Figure 2 The position of probe and the sonogram for the modified QL block below the LAL. (A) the position of probe. The yellow line shows the position of LAL. The white rectangular box shows the position of probe for the modified subcostal QL block below the LAL. The blue rectangular box shows the position of probe for the classic subcostal QL block. (B) the sonogram showing the sonoanatomic relationship among diaphragm, LAL and the ATF. The diaphragm appeared hypoechoic and was sandwiched between two bright echogenic lines of fascia. The lowest edge of the diaphragm was considered as the LAL, which could be seen when shifting the probe laterally to the tip of LI TP. The LAL is in continuity with the ATF. The QL muscle is lined with the diaphragm at the lateral supra-arcuate ligament, and with the ATF below the LAL. Abbreviations: TP, transverse process; QL, quadratus lumborum; LAL, lateral arcuate ligament; ATF, anterior thoracolumbar fascia.

hematoma, renal injury and pneumothorax by using ultrasound scan.

\section{Results}

One patient was excluded because the diaphragm and ATF were not clearly visualized on the ultrasound image during the ultrasound scout scan. 12 men and 11 women with a mean age of 63.1 (6.4) yr, weight 65.8 (3.5) kg, height $163.2(3.9) \mathrm{cm}$, and BMI 25.8 (3.7) received the modified block. The ultrasound scout scan was successful in 23 out of $24(95.8 \%)$ patients, in whom the QL muscle, the diaphragm and the ATF were visualized.

After sterile preparation, the total ultrasound visibility of QL muscle, diaphragm and ATF was judged as good in 19 out of $23(82.6 \%)$, and average in 4 out of $23(17.4 \%)$ patients. The total ultrasound visibility score of the QL muscle, diaphragm and ATF in this cohort of patients was 8 (range 5-9). The diaphragm appeared hypoechoic and was sandwiched between two bright echogenic lines of fascia. ${ }^{9}$ With inspiration, the diaphragm moved caudally. The lowest edge of the diaphragm was considered as the lateral arcuate ligament. ${ }^{8,9}$ The continuity of the lateral arcuate ligament with the ATF also could be observed in the sonogram (Figure 2B and Video 1). The QL muscle was shown as the hypoechoic or isoechoic structure posterior to the diaphragm (Figure 2B).

The modified subcostal QL block below the lateral arcuate ligament was successfully performed in 23 patients with successful scout scans, in which 1 (range 1-3) attempt was taken. During the injection, the apparent downward displacement of the diaphragm and the ATF was observed in these 23 patients, indicating the spread of LA in the posterior pathway of lateral arcuate ligament (Figure $3 \mathrm{~A}$ and Video 2). The average procedure duration was $78 \pm 5$ seconds, which is acceptable in clinical practice.

The paramedian transverse scanning at the level of T12-L1 was performed immediately after the block to observe whether the LA injectate spread into the T12 paravertebral space. The lunar-shaped spread of LA along the diaphragm into the T12 paravertebral space was observed in all patients (Figure 3B).

At 5 min after injection, the sensory blockade range from T9-T12 was obtained in every patient, while the sensory blockade range from T6-L1 was available in every patient at $10 \mathrm{~min}$ after injection. 2 out of 23 (8.7\%) patients achieved coverage as cephalad as $\mathrm{T} 5$. The mean numbers of block dermatomes were $5.6 \pm 1.8$ at $5 \mathrm{~min}$ and $6.5 \pm 2.2$ at $10 \mathrm{~min}$ after injection.

No pneumothorax and renal injury occurred in all patients. All patients recovered from this block without other complications.

\section{Discussion}

In the study, we have shown the successful application of the modified QL block below the lateral arcuate ligament, in which the needle was inserted in the plane of the ultrasound beam. It reliably provides the sensory blockade to nearly the entire abdomen.

Elsharkawy et al developed the classic subcostal QL block. ${ }^{1}$ This block targets the LA between the investing fascia of the QL muscle and the ATF at L1-2 level with the 

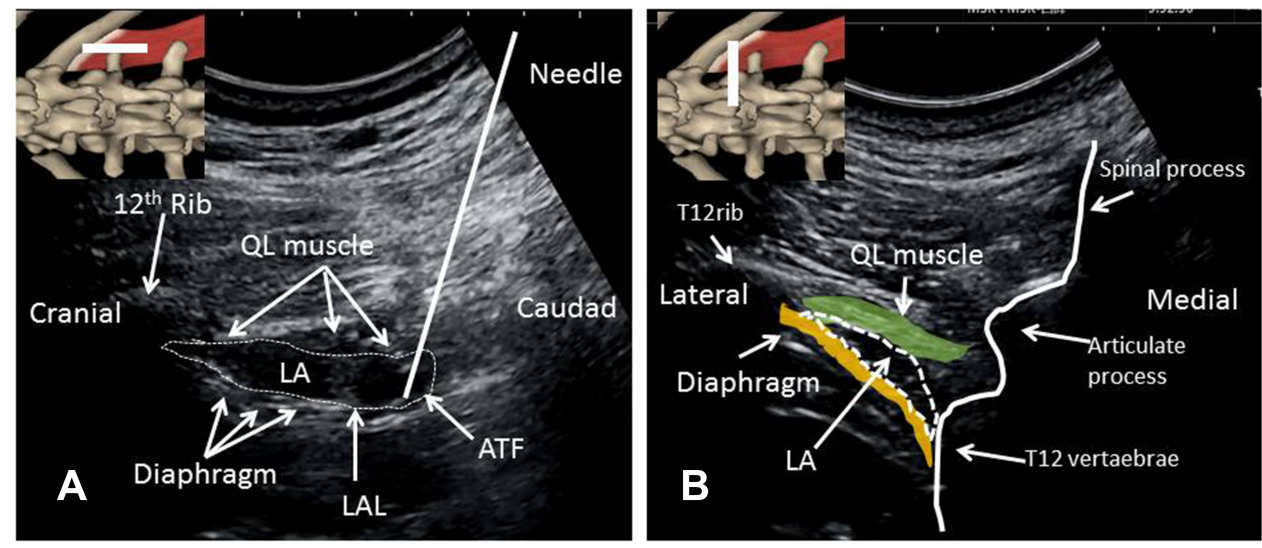

Figure 3 The sonograms showing the local anesthetic spread under paramedian sagittal view and paramedian transverse view. (A) Paramedian sagittal sonogram during realtime ultrasound-guided modified QL block. The target injection site is below the LAL and between the investing fascia of QL muscle and the ATF. During the injection, the apparent downward displacement of diaphragm and the ATF was observed. (B) The lunar-shaped spread of LA along the diaphragm into the TI2 paravertebral space was observed under paramedian transverse view at the level of TI2-LI immediately after injection.

Abbreviations: QL, quadratus lumborum; LAL, lateral arcuate ligament; LA, local anesthetic; ATF, anterior thoracolumbar fascia.

transducer directed cranially, 6-8 $\mathrm{cm}$ lateral to the L1 spinous process and angled medially (Figure 2A). It can provide a sensory block with dermatomal coverage from T6-7 to L1-2. ${ }^{10}$ The subcostal QL block allows LA to spread cranially via a posterior pathway of the lateral arcuate ligament and into the lower thoracic paravertebral space posterior to the endothoracic fascia, which was validated in a cadaver study. ${ }^{2}$ So, the lateral arcuate ligament and its posterior pathway are the key structures for understanding the action mechanism of classic subcostal QL block. However, Dr. Elsharkawy did not describe the ultrasonography of the lateral arcuate ligament and its posterior pathway in his technique. In his approach, the probe is placed too laterally to clearly visualize the diaphragm and lateral arcuate ligament. In the current study, our group developed a method to identify the sonoanatomy of the lateral arcuate ligament and its posterior pathway. Further, we modified the classic subcostal QL block. In our modified QL block, the cranial spread of LA via the posterior pathway of the lateral arcuate ligament was considered as an indicator of successful injection.

In the current study, the ultrasound scout scan for visualizing QL and diaphragm is successful in most patients $(95.8 \%)$. The total ultrasound visibility of QL muscle and diaphragm was judged as good in $82.6 \%$ and average in $17.4 \%$ patients after sterile preparation. The high imaging quality might be associated with the lower average weight $(65.8 \pm 3.5 \mathrm{~kg})$ in this cohort of patients, since excessive fat in obesity may decrease image quality by attenuating the transmission of the US signal, increasing the overall depth to the target structures. ${ }^{6}$ Future studies should evaluate the utility of ultrasound guidance in the obese since the modified technique in these patients may be challenging.

During the injection, the apparent downward displacement of the diaphragm and the ATF was observed in these 23 patients, indicating the spread of LA into the posterior pathway via the lateral arcuate ligament. The number of 1 (range 1-3) attempt was taken for the modified QL block. In the current study, the procedure time was defined from the scanning of structures after sterile preparation to the time of LA injection completion, excluded time to assess the structures visibility. The average procedure duration was 78 seconds. Since the block needle was inserted in the plane of the ultrasound beam, it was possible to visualize the advancing needle in real time. It may help reduce the number of attempts and the time for accessing the target compartment.

The paramedian transverse scanning at the level of T12-L1 was performed to monitor whether the LA spread into the T12 paravertebral space immediately after the modified QL block. The transverse ultrasound images showed the lunar-shaped spread of LA along the diaphragm into the T12 paravertebral space, which indicates that the action mechanism of the modified QL block is the LA spread into the lower thoracic paravertebral space from the injection site. ${ }^{11,12}$

The dermatomal coverage of sensory block with the modified QL block is similar to that with the subcostal QL block. The innervation of the abdominal wall is derived from 
the thoracic level of T6-L1, so blockade of these sensory dermatomes with the new approach should provide the analgesic efficacy in abdominal surgery. ${ }^{10,13}$ The transmuscular QL block is reported to generate analgesia from T10L4, which is not suitable for upper abdominal surgery. ${ }^{14,15}$

The block onset time depends on the type and concentration of local anesthetic, and the frequency of assessment. Borglum et $\mathrm{al}^{16}$ have demonstrated that the transmuscular QL block is characterized by a $30 \mathrm{~min}$ block onset time with $30 \mathrm{~mL}$ of $0.375 \%$ ropivacaine. Zhu et $\mathrm{al}^{17}$ have reported that the T6-L1 dermatomal coverage could be achieved at $30 \mathrm{~min}$ after subcostal QL bock $(0.3 \%$ ropivacaine, $0.4 \mathrm{~mL} / \mathrm{kg})$. Lu et $\mathrm{al}^{18}$ have also tested the area of sensory block at 30 min after transmuscular QL block based on the report on the onset time from Borglum et al. In our current study, $20 \mathrm{~mL}$ of $0.5 \%$ ropivacaine was used for the modified block based on our study on the thoracic paravertebral block. ${ }^{19}$ The sensory block with the modified QL block could be tested in all patients at $5 \mathrm{~min}$ after LA injection, indicating the rapid onset with the new block when $20 \mathrm{~mL}$ of $0.5 \%$ ropivacaine was given. It may be attributed to the spread of LA into the lower thoracic paravertebral space via the posterior pathway of lateral arcuate ligament and the higher ropivacaine concentration used. The rapid block onset time can help anesthetists judge whether the block is successful or not and in time optimize the intraoperative anesthesia management or postoperative analgesia strategy. Future comparisons between this technique and others on the onset time with the same type and concentration of local anesthetic are required.

The ultrasound-guided transmuscular QL block catheter technique has been reported by Kadam et al. ${ }^{20}$ The catheter technique can prolong the block action of QL block and may be beneficial for postoperative recovery. For our modified block, the sagittal ultrasound scan was used and the apparent downward displacement of the diaphragm and the ATF was observed after the injection. It indicates that the catheter technique is easily performed in this new block. The future investigation needs to be done to make sure the effectiveness of the modified QL block catheter technique.

The injection target site is below the lateral arcuate ligament and the pneumothorax could be avoided by distinguishing diaphragm from bright echogenic pleura. No case developed pneumothorax in the current study. Similarly, the renal injury associated did not occur in this cohort of patients, since the block needle trajectory was clearly visualized with the needle in the plane of ultrasound beam, and the total ultrasound visibility of target structures was judged as good and average.

The current study has several limitations. We only included the patients with $\mathrm{BMI}<28$. Future studies should evaluate the utility of the technique we described above in the obese patients. Another limitation of our study is that it is descriptive and not a randomized trial. Larger randomized trials are required to quantify the success and failure rates of the technique described. Additionally, the comparisons between this technique and classic QL blocks with comprehensive measures of success, including the pain scores after operation and postoperative opioid consumption are required to establish the role of this block in improving the surgical outcomes in the future.

\section{Conclusions}

In summary, we have demonstrated the successful use of the modified QL block below the lateral arcuate ligament, which is characterized as rapid onset time $(<5 \mathrm{~min})$, consistent block segments, and high success rate. Further randomized trials are required to establish the role of this block in improving the surgical outcomes.

\section{Data Sharing Statement}

The corresponding author (Dr. Yun Wang, wangyun129@ccmu.edu.cn)will be ready to share all related data for any authority upon request by an email message. The data will be available this way for up to 6 months after the paper is published.

\section{Acknowledgments}

This work was supported by the Beijing Hospitals Authority Clinical Medicine Development of Special Funding Support (XMLX202106).

\section{Disclosure}

The authors declare no conflicts of interest.

\section{References}

1. Elsharkawy H. Quadratus lumborum block with paramedian sagittal oblique (subcostal) approach. Anaesthesia. 2016;71:241-242.

2. Elsharkawy H, El-Boghdadly K, Kolli S, et al. Injectate spread following anterior sub-costal and posterior approaches to the quadratus lumborum block: a comparative cadaveric study. Eur J Anaesthesiol. 2017;34:587-595.

3. Willard FH, Vleeming A, Schuenke MD, Danneels L, Schleip R. The thoracolumbar fascia: anatomy, function and clinical considerations. J Anat. 2012;221:507-536. 
4. Li H, Ma D, Liu Y, Wang Y. A transverse approach for ultrasound-guided anterior quadratus lumborum block at the lateral supra-arcuate ligament. Anaesthesia. 2020;75:1400-1401.

5. Kanemura T, Satake K, Nakashima H, Naoki S, Ouchida J, Yamaguchi H. Understanding retroperitoneal anatomy for lateral approach spine surgery. Spinal Surg Rel Res. 2017;1:107-120.

6. Karmakar MK, Li X, Ho AM, Kwok WH, Chui PT. Real-time ultrasound-guided paramedian epidural access: evaluation of a novel in-plane technique. Br J Anaesth. 2009;102:845-854.

7. Li H, Kang Y, Jin L, Ma D, Liu Y, Wang Y. Feasibility of ultrasound-guided lumbar epidural access using paramedian transverse scanning with the needle in-plane: a comparison with paramedian sagittal scanning. J Anesth. 2020;34(1):29-35.

8. Shi R, Li H, Wang Y. Dermatomal coverage of single-injection ultrasound-guided parasagittal approach to anterior quadratus lumborum block at the lateral supra-arcuate ligament. J Anesth. 2021. doi:10.1007/s00540-021-02903-1

9. Troyer AD, Wilson TA. Action of the diaphragm on the rib cage. J Appl Physiol (1985). 2016;121:391-400.

10. Elsharkawy H, Ahuja S, DeGrande S, Maheshwari K, Chan V. Subcostal approach to anterior quadratus lumborum block for pain control following open urological procedures. $J$ Anesth. 2019;33:148-154.

11. Tamura T, Yokota S, Ito S, Shibata Y, Nishiwaki K. Local anesthetic spread into the paravertebral space with two types of quadratus lumborum blocks: a crossover volunteer study. $J$ Anesth. 2019;33:26-32.

12. Elsharkawy H, El-Boghdadly K, Barrington M. Quadratus lumborum block: anatomical concepts, mechanisms, and techniques. Anesthesiology. 2019;130:322-335.
13. Okmen K, Metin OB, Topal S. Ultrasound-guided posterior quadratus lumborum block for postoperative pain after laparoscopic cholecystectomy: a randomized controlled double blind study. J Clin Anesth. 2018;49:112-117.

14. Kukreja P, MacBeth L, Sturdivant A, et al. Anterior quadratus lumborum block analgesia for total hip arthroplasty: a randomized, controlled study. Reg Anesth Pain Med. 2019;44:1075-1079.

15. Kinjo S, Kolodzie K, Dong K, Zhang AL. The effects of transmuscular quadratus lumborum blocks on postoperative pain in arthroscopic hip surgery: a cohort analysis. J Anesth. 2019;33:516-522.

16. Borglum J, Moriggl B, Jensen K, et al. Ultrasound-guided transmuscular quadratus lumborum blockade. Br J Anaesth. 2013;111.

17. Zhe M, Qi Y, He H, Lou J, Pei Q, Mei Y. Analgesic effect of the ultrasound-guided subcostal approach to transmuscular quadratus lumborum block in patients undergoing laparoscopic nephrectomy: a randomized controlled trial. BMC Anesthesiol. 2019;19:154.

18. Lu Y, Zhang J, Xu X, et al. Sensory assessment and block duration of transmuscular quadratus lumborum block at L2 versus L4 in volunteers: a randomized controlled trial. Minerva Anestesiol. 2019;85:1273-1280.

19. Li H, Wei H, Ma D, Wang Y. Ultrasound and pressure-guided thoracic paravertebral block, A preliminary investigation. Eur $J$ Anaesthesiol. 2020;37:824-835.

20. Kadam VR, Ludbrook G, Van Wijk RM, et al. Comparison of ultrasound-guided transmuscular quadratus lumborum block catheter technique with surgical preperitoneal catheter for postoperative analgesia in abdominal surgery: a randomized controlled trial. Anaesthesia. 2019;74:1381-1388.
Journal of Pain Research

\section{Publish your work in this journal}

The Journal of Pain Research is an international, peer reviewed, open access, online journal that welcomes laboratory and clinical findings in the fields of pain research and the prevention and management of pain. Original research, reviews, symposium reports, hypothesis formation and commentaries are all considered for publication. The manuscript management system is completely online and includes a very quick and fair peer-review system, which is all easy to use. Visit http:// www.dovepress.com/testimonials.php to read real quotes from published authors. 